\title{
Platelet Count is Associated with the Rate of Lymph Node Metastasis in Lung Adenocarcinoma
}

This article was published in the following Dove Press journal:

Cancer Management and Research

\author{
Cheng-hao $\mathrm{Qu}{ }^{1,2}$ \\ Tong $\mathrm{Li}^{1,2}$ \\ Zhan-peng Tang ${ }^{1,2}$ \\ Xi-Rui Zhu (D) ${ }^{1,2}$ \\ Jing-yi $\operatorname{Han}^{1,2}$ \\ Hui Tian ${(\mathbb{D})^{2}}^{2}$ \\ 'Cheeloo College of Medicine, Shandong \\ University, Jinan, Shandong 2500I2, \\ People's Republic of China; ${ }^{2}$ Department \\ of Thoracic Surgery, Qilu Hospital, \\ Cheeloo College of Medicine, Shandong \\ University, Jinan, Shandong 2500I2, \\ People's Republic of China
}

Correspondence: Hui Tian Department of Thoracic Surgery, Qilu Hospital, Cheeloo College of Medicine, Shandong University, Jinan, Shandong 2500 I2, People's Republic of China

Tel +8618560080016

Email tianhuiq|@I26.com
Purpose: Emerging studies have revealed that platelets are involved in tumor metastasis in lung adenocarcinoma (ADC). The solid pathological subtype of lung ADC is associated with metastasis, recurrence, and poor prognosis. However, there is no study exploring the relationship between platelets and different lung pathological subtypes.

Patients and Methods: The association between platelet counts and lymph node metastasis was analyzed in 852 patients with lung ADC who underwent surgery and lymph node dissection. Multivariate logistic analysis was conducted to identify the risk factors of lymph node metastasis. Then, lymph node metastasis and other factors were analyzed to determine their correlation with platelet count and histological subtype.

Results: We found that the platelet count was associated with lymph node metastasis $(\mathrm{P}=0.01)$ in multivariable analysis, independent of tumor size, predominant subtype, visceral pleural invasion, and microvessel invasion. In patients with a platelet count $\geq 300 \times 109 / \mathrm{L}$, the rate of lymph node metastasis was $38.5 \%$, almost twice as high as that in patients with a platelet count $<300 \times 109 / \mathrm{L}(23.2 \%)$. Additionally, elevated platelet counts, even those within the normal range, were significantly associated with a higher rate of lymph node metastasis. The mean platelet count in patients with solid-predominant histology $(269.70 \pm 69.38 \times 109 / \mathrm{L})$ was significantly higher than that in patients with other histologies $(\mathrm{P}<0.001)$.

Conclusion: Elevated platelet counts are significantly associated with a higher rate of lymph node metastasis, even if the platelet counts are within the reference range. Platelet counts were significantly higher in patients with solid-predominant histology than in patients with other histologies. In addition, VEGF-C may play an important role in lymphatic metastasis in patients with lung ADC. We hypothesize that antiplatelet therapy may reduce lymph node metastasis in lung ADC patients.

Keywords: lung adenocarcinoma, platelets, lymph node, solid-predominant, pathological subtype

\section{Introduction}

Lung cancer is the most commonly diagnosed cancer $(11.6 \%$ of all cancer diagnoses) and the leading cause of cancer-related death (18.4\% of all cancerrelated deaths) in both men and women. ${ }^{1}$ Lung adenocarcinoma (ADC) is an independent pathologic subtype of lung cancer that has been widely studied. In 2011, the International Association for the Study of Lung Cancer, American Thoracic Society, and European Respiratory Society (IASLC/ATS/ERS) proposed a new histologic classification of lung ADC. ${ }^{2}$ Invasive lung ADC was divided into six major pathological subtypes: lepidic, acinar, papillary, micropapillary, solid, and invasive mucinous adenocarcinoma. Several studies have reported that 
the micropapillary and solid subtypes of lung ADC are associated with metastasis, recurrence, and poor prognosis. $^{3-7}$ However, the reason for this phenomenon is unclear.

Emerging research suggests that platelets are functional players in many steps of the metastatic process. ${ }^{8-11}$ It is clear that cancer cells can induce abnormalities in platelet number and function. In turn, platelets can promote tumor metastasis. $^{12,13}$ Tumor-educated platelets (TEPs) may be an ideal ally of tumor progression. ${ }^{14}$ Conversely, platelets can be continuously activated and augment cancerassociated thrombosis. ${ }^{15}$ Elevated platelet counts have been observed in patients with advanced cancer affecting the breast, colon, gastric, lung, ovary, and esophagus. ${ }^{16-22}$ Further, elevated platelet counts are sometimes related to poor prognosis. ${ }^{16-22}$ Although there is still some controversy in breast cancer, most preclinical studies indicate an association between elevated platelets and metastasis, ${ }^{23}$ as well as with lymph node metastasis, and poor prognosis in lung cancer. ${ }^{24,25}$

Lung ADC metastasis is mainly classified into hematogenous and lymphatic metastases. Previous studies have mainly reported that preoperative elevated platelet counts have a role in promoting hematogenous metastasis and predicting a poor prognosis in non-small cell lung cancer patients, ${ }^{23,24}$ the relationships between platelets, lymph node metastasis, and different ADC subtypes have not been widely studied. We conducted this study to explore the relationship of platelets, lymph node metastasis, and different ADC subtypes.

\section{Patients and Methods}

\section{Ethical Statement and Patients}

This retrospective study has been approved bythe institutional review board of the Qilu Hospital of Shandong University (KYLL-2016-097). All patients provided informed consent for the use of their clinical information. One thousand four hundred and seventy-two patients underwent surgical resection for pathologically diagnosed invasive lung ADC between January 2013 and December 2016 in the Qilu Hospital. All cases were collected through the medical record information database of the Qilu Hospital of Shandong University. The inclusion criteria were as follows: (1) patients underwent surgery for pathologically confirmed lung invasive $\mathrm{ADC}$, and the pathological subtypes were recorded according to the 2011 IASLC/ATS/ERS standard; ${ }^{2}$ (2) patients underwent computed tomography, magnetic resonance imaging, bone scans, and positron emission tomography in accordance with the recommendations of the Chinese Society of Clinical Oncology guidelines within 14 days before surgery; (3) no distant metastasis or other malignancies; (4) no neoadjuvant chemotherapy or radiotherapy; (5) no hematological diseases before surgery; and (6) no antiplatelet drugs such as aspirin. After screening, a total of 852 patients were eligible for inclusion.

\section{Blood Samples}

All patients underwent routine blood testing in our hospital before surgery. Blood was collected in a blood collection tube containing EDTA for anticoagulation between 6:00 and 8:00 in the morning when patients were in the fasting state, and blood was sent for examination within 2 hours.

\section{Pathological Examination}

After surgery, all excised specimens were fixed in formalin and immediately stained with hematoxylin and eosin, with at least two cut surfaces of lymph nodes and three cut surfaces of tumor tissue being stained. Microscopic evaluation was performed by two pathologists, and if there were differences in the results, no less than two additional pathologists discussed the case to reach an agreement. Pathologists adopted the newly announced IASLC/ATS/ ERS classification system for lung ADC. Since most lung ADCs fall into the mixed subtype, comprehensive histologic subtyping has been proposed to make a semiquantitative assessment of the percentages of the various histologic components and to classify tumors according to the predominant histologic subtype. ${ }^{2}$ The percentage of each histological component was recorded in 5\% increments. The predominant subtype was defined as the subtype with the largest percentage, even if it accounted for less than $50 \%$ of the tumor. Minor components referred to subtypes that occupied more than $5 \%$ of the tumor but were not the predominant subtype. The presence of visceral pleural invasion (VPI), ${ }^{26}$ microvessel invasion (MVI), ${ }^{27,28}$ and spread through air spaces (STAS) were also determined. ${ }^{29,30}$

\section{Statistical Analysis}

Clinical and pathological characteristics, such as gender, age, smoking status, platelet count, platelet groups, tumor location, surgery, predominant subtype, tumor diameter, T stage, STAS, VPI, and MVI were analyzed to determine their correlation with lymph node metastasis. Continuous random variables, such as platelet count, age, and tumor 
diameter, were expressed as mean \pm standard deviation (SD) or mean \pm standard error of mean (SEM) and analyzed using the independent samples $t$-test. On univariate analysis, the effect of each factor on the probability of lymph node metastasis and MVI was assessed based on the logistic regression model. All variables with $\mathrm{P}<0.1$ in the univariate analysis were entered into the multivariate analysis. On multivariate analysis, the stepwise logistic regression analysis was conducted by utilizing these factors. Odds ratios (ORs) and 95\% confidence intervals (CIs) were reported for both univariate and multivariate analyses. Lymph node metastasis, STAS, VPI, and MVI were analyzed to determine the correlation with predominant subtypes, the solid-predominant/minor components subtype, and the platelet count. $\mathrm{P}$ values were calculated using Pearson's Chi-square test or Fisher's exact test, and the significance level was set at 0.05 . The data were analyzed using IBM SPSS Statistics, version 25.0 (IBM, USA).

\section{Results}

The characteristics of all 852 patients are listed in Table 1. We divided patients based on $\mathrm{N}$ stage: 635 (74.5\%) had pathologically validated N0 disease and 217 (25.5\%) had validated $\mathrm{N} 1$ or $\mathrm{N} 2$ disease. No significant differences were observed in terms of gender, age, smoking history, or STAS between the two groups. Platelet count, tumor location, surgical method, predominant subtype, pathological T stage, VPI, and MVI were significantly different between the two groups.

\section{Risk Factors of Lymph Node Metastasis}

We first performed univariate regression analysis of variables predicting lymph node metastasis (Table 2). In univariate analysis, platelet count, tumor location, surgical method, tumor size, predominant subtype, VPI, and MVI were significantly associated with lymph node metastasis $(\mathrm{P}<0.05)$. In multivariate analysis, we found that platelet count $(\mathrm{aOR}=$ $1.004,95 \% \mathrm{CI}=1.001-1.007 ; \mathrm{P}=0.010)$, tumor size $(\mathrm{aOR}=$ $1.699,95 \% \mathrm{CI}=1.471-1.963 ; \mathrm{P}<0.001), \mathrm{VPI}(\mathrm{aOR}=1.635$, 95\% $\mathrm{CI}=1.053-22.332 ; \mathrm{P}=0.029)$, and $\mathrm{MVI}(\mathrm{aOR}=3.074$, $95 \% \mathrm{CI}=1.773-5.330 ; \mathrm{P}<0.001)$ remained independent risk factors for lymph node metastasis. The predominant subtype was also a significant independent risk factor of lymph node metastasis. Compared to patients with the lepidic subtype, patients with the acinar subtype had an aOR of $10.430(95 \%$ CI $=3.693-29.457, \mathrm{P}<0.001)$, patients with
Table I Clinicopathological Characteristics of Different Lymph Node Status in 852 Patients with Lung Adenocarcinoma

\begin{tabular}{|c|c|c|c|c|}
\hline & $\begin{array}{l}\text { Total } \\
(n=852)\end{array}$ & $\begin{array}{l}\text { No } \\
(n=635)\end{array}$ & $\begin{array}{l}N 1 / 2 \\
(n=217)\end{array}$ & $P$ value \\
\hline $\begin{array}{l}\text { Gender } \\
\text { Male } \\
\text { Female }\end{array}$ & $\begin{array}{l}392(46) \\
460(54)\end{array}$ & $\begin{array}{l}283(44.6) \\
352(55.4)\end{array}$ & $\begin{array}{l}109(50.2) \\
108(49.8)\end{array}$ & $0.148^{\mathrm{a}}$ \\
\hline Age (year) & & $\begin{array}{l}59.77 \\
\pm 9.376\end{array}$ & $\begin{array}{l}59.53 \\
\pm 9.870\end{array}$ & 0.745 \\
\hline $\begin{array}{l}\text { Smoking } \\
\text { Current or ex } \\
\text { Never }\end{array}$ & $\begin{array}{l}277(32.5) \\
575(67.5)\end{array}$ & $\begin{array}{l}199(31.3) \\
436(68.7)\end{array}$ & $\begin{array}{l}78(35.9) \\
139(64.1)\end{array}$ & 0.211 \\
\hline PLT (×10^9/L) & & $\begin{array}{l}235.57 \\
\pm 58.390\end{array}$ & $\begin{array}{l}258.59 \\
\pm 62.733\end{array}$ & $0.000^{\mathrm{a}}$ \\
\hline $\begin{array}{l}\text { Tumor location } \\
\text { LUL } \\
\text { LLL } \\
\text { RUL } \\
\text { RML } \\
\text { RLL }\end{array}$ & $\begin{array}{l}200(23.5) \\
140(16.4) \\
278(32.6) \\
57(6.7) \\
123(19.4)\end{array}$ & $\begin{array}{l}143(22.5) \\
98(15.4) \\
226(35.6) \\
45(7.1) \\
54(24.9)\end{array}$ & $\begin{array}{l}57(26.3) \\
42(19.4) \\
52(24) \\
12(5.5) \\
177(20.8)\end{array}$ & $0.016^{a}$ \\
\hline $\begin{array}{l}\text { Surgery } \\
\text { Lobectomy } \\
\text { Limited resection } \\
\text { Pneumonectomy }\end{array}$ & $\begin{array}{l}792(93) \\
54(6.3) \\
6(0.7)\end{array}$ & $\begin{array}{l}593(93.4) \\
4 I(6.5) \\
I(0.2)\end{array}$ & $\begin{array}{l}199(91.7) \\
13(6.0) \\
5(2.3)\end{array}$ & $0.012^{\mathrm{a}, \mathrm{b}}$ \\
\hline $\begin{array}{l}\text { Predominant subtype } \\
\text { L } \\
\text { A } \\
\text { P } \\
\text { MP } \\
\text { S } \\
\text { IMA }\end{array}$ & $\begin{array}{l}148(17.4) \\
426(50) \\
129(15.1) \\
23(2.7) \\
86(10.1) \\
40(4.7)\end{array}$ & $\begin{array}{l}144(22.7) \\
317(49.9) \\
100(15.7) \\
6(0.9) \\
39(6.1) \\
29(4.6)\end{array}$ & $\begin{array}{l}4(1.8) \\
109(50.2) \\
29(13.4) \\
17(7.8) \\
47(21.7) \\
11(5.1)\end{array}$ & $0.000^{\mathrm{a}}$ \\
\hline Tumor size $(\mathrm{cm})$ & & $\begin{array}{l}2.267 \\
\pm 1.118\end{array}$ & $\begin{array}{l}3.514 \\
\pm 1.785\end{array}$ & $0.000^{\mathrm{a}}$ \\
\hline $\begin{array}{l}\text { T stage } \\
\text { T1 } \\
\text { T2 } \\
\text { T3/T4 }\end{array}$ & $\begin{array}{l}663(77.8) \\
147(17.3) \\
42(4.9)\end{array}$ & $\begin{array}{l}546(86.0) \\
76(12) \\
29(2.0)\end{array}$ & $\begin{array}{l}117(53.9) \\
71(32.7) \\
13(13.4)\end{array}$ & $0.000^{\mathrm{a}}$ \\
\hline $\begin{array}{l}\text { STAS } \\
\text { Absent } \\
\text { Present }\end{array}$ & $\begin{array}{l}765(89.8) \\
87(10.2)\end{array}$ & $\begin{array}{l}570(89.8) \\
65(10.2)\end{array}$ & $\begin{array}{l}195(89.9) \\
22(10.1)\end{array}$ & 0.967 \\
\hline $\begin{array}{l}\text { VPI } \\
\text { Absent } \\
\text { Present }\end{array}$ & $\begin{array}{l}708(83.1) \\
144(16.9)\end{array}$ & $\begin{array}{l}547(86.1) \\
88(13.9)\end{array}$ & $\begin{array}{l}161(74.2) \\
56(25.8)\end{array}$ & $0.000^{\mathrm{a}}$ \\
\hline $\begin{array}{l}\text { MVI } \\
\text { Absent } \\
\text { Present }\end{array}$ & $\begin{array}{l}773(90.7) \\
79(9.3)\end{array}$ & $\begin{array}{l}603(95) \\
32(5)\end{array}$ & $\begin{array}{l}170(78.3) \\
47(21.7)\end{array}$ & $0.000^{\mathrm{a}}$ \\
\hline
\end{tabular}

Notes: Data are presented as $n(\%)$ or means \pm SD. P value, and OR were calculated using the Chi-square test and Fisher's exact test. ${ }^{a}$ Statistically significant. ${ }^{b}$ Fisher's exact test. Abbreviations: OR, odds ratio; LUL, left upper lobe; LLL, left lower lobe; RUL, right upper lobe; RML, right middle lobe; RLL, right lower lobe; L, lepidic; A, acinar; P, papillary; MP, micropapillary; S, solid; IMA, invasive mucinous adenocarcinoma; STAS, spread through air spaces; VPI, visceral pleural invasion; MVI, microscopic vessel invasion. 
Table 2 Univariate and Multivariate Logistic Analyses of the Risk Factors Associated with Lymph Nodes Metastasis

\begin{tabular}{|c|c|c|c|c|c|c|}
\hline \multirow[t]{2}{*}{ Variables } & \multicolumn{3}{|c|}{ Univariate Analysis } & \multicolumn{3}{|c|}{ Multivariate Analysis } \\
\hline & OR & $95 \% \mathrm{Cl}$ & $P$ value & aOR & $95 \% \mathrm{Cl}$ & $P$ value \\
\hline \multicolumn{7}{|l|}{ Gender } \\
\hline Male & 1 & & & & & \\
\hline Female & 0.797 & $0.585-1.085$ & 0.149 & & & \\
\hline Age (year) & 0.997 & $0.981-1.014$ & 0.745 & & & \\
\hline \multicolumn{7}{|l|}{ Smoking } \\
\hline Current or ex & 1 & & & & & \\
\hline Never & 0.813 & $0.588-1.125$ & 0.211 & & & \\
\hline PLT (×10^9/L) & 1.006 & $1.004-1.009$ & $0.000^{\mathrm{a}}$ & 1.004 & $1.001-1.007$ & $0.010^{\mathrm{a}}$ \\
\hline \multicolumn{7}{|l|}{ Tumor location } \\
\hline LUL & I & & & & & \\
\hline LLL & 1.075 & $0.669-1.728$ & 0.764 & & & \\
\hline RUL & 0.577 & $0.375-0.888$ & $0.012^{\mathrm{a}}$ & & & \\
\hline RML & 0.669 & $0.330-1.357$ & 0.265 & & & \\
\hline RLL & 1.101 & $0.707-1.716$ & 0.669 & & & \\
\hline \multicolumn{7}{|l|}{ Surgery } \\
\hline Limited resection & I & & & & & \\
\hline Lobectomy & 1.058 & $0.556-2.016$ & 0.863 & & & \\
\hline Pneumonectomy & 15.769 & $1.686-147.509$ & $0.016^{\mathrm{a}}$ & & & \\
\hline Tumor size $(\mathrm{cm})$ & 1.921 & $1.678-2.200$ & $0.000^{\mathrm{a}}$ & 1.699 & $1.47 \mathrm{I}-1.963$ & $0.000^{\mathrm{a}}$ \\
\hline \multicolumn{7}{|l|}{ Predominant subtype } \\
\hline $\mathrm{L}$ & I & & & 1 & & \\
\hline$A$ & 12.379 & $4.477-34.227$ & $0.000^{\mathrm{a}}$ & 10.430 & $3.693-29.457$ & $0.000^{\mathrm{a}}$ \\
\hline$P$ & 10.440 & $3.559-30.622$ & $0.000^{\mathrm{a}}$ & 6.779 & $2.228-20.633$ & $0.00 I^{\mathrm{a}}$ \\
\hline$M P$ & 102.000 & $26.144-397.946$ & $0.000^{\mathrm{a}}$ & 68.016 & $16.734-276.455$ & $0.000^{\mathrm{a}}$ \\
\hline S & 43.385 & |4.727-|27.805 & $0.000^{\mathrm{a}}$ & 19.235 & $6.262-59.082$ & $0.000^{\mathrm{a}}$ \\
\hline IMA & 13.655 & $4.064-45.880$ & $0.000^{\mathrm{a}}$ & 5.952 & I.587-22.332 & $0.008^{\mathrm{a}}$ \\
\hline \multicolumn{7}{|l|}{ STAS } \\
\hline Absent & I & & & & & \\
\hline Present & 0.989 & $0.594-1.648$ & 0.967 & & & \\
\hline \multicolumn{7}{|l|}{ VPI } \\
\hline Absent & I & & & 1 & & \\
\hline Present & 2.162 & $1.48 I-3.156$ & $0.000^{\mathrm{a}}$ & 1.635 & I.053-22.332 & $0.029^{\mathrm{a}}$ \\
\hline \multicolumn{7}{|l|}{ MVI } \\
\hline Absent & 1 & & & 1 & & \\
\hline Present & 5.210 & $3.223-8.422$ & $0.000^{\mathrm{a}}$ & 3.074 & $1.773-5.330$ & $0.000^{\mathrm{a}}$ \\
\hline
\end{tabular}

Notes: $\mathrm{P}$ value, $\mathrm{OR}$, and $95 \% \mathrm{Cl}$ were calculated using the Chi-square test and Fisher's exact test. aOR, adjusted odds ratio. ${ }^{a}$ Statistically significant. Abbreviation: $\mathrm{Cl}$, confidence interval.

the papillary subtype had an aOR of $6.779(95 \% \mathrm{CI}=$ 2.228-20.633, $\mathrm{P}=0.001$ ), patients with the micropapillary subtype had an aOR of 68.016 (95\% CI $=16.734-276.455$, $\mathrm{P}<0.001$ ), patients with the solid subtype had an aOR of 19.235 (95\% CI $=6.262-59.082, \mathrm{P}<0.001)$, and patients with invasive mucinous ADC had an aOR of 5.952 (95\% CI $=1.587-22.332, \mathrm{P}=0.008)($ Table 2$)$.

\section{The Influence of Platelet Count on Tumor}

\section{Behavior}

To determine the effect of platelet count on tumor behavior, we divided the patients into the reference range platelet count group (group $\alpha$ : platelet count $\left.100-300 \times 10^{9} / \mathrm{L}\right)$ and the thrombocytosis group (group $\beta$ : platelet count $\geq 300 \times 10^{9} / \mathrm{L}$ ). There were 
Table 3 The Influence Between Normal Range Platelet Count (Group $\alpha$ ) and Thrombocytosis (Group $\beta$ ) on Microscopic Vessel Invasion (MVI) and Lymph Nodes Metastasis

\begin{tabular}{|c|c|c|c|}
\hline PLT Group & $\alpha(720)$ & $\beta(130)$ & $P$ value \\
\hline T stage, $\mathrm{n}(\%)$ & & & $0.019^{a}$ \\
\hline TI & $570(79.2)$ & $91(70)$ & \\
\hline $\mathrm{T} 2$ & $120(16.7)$ & $27(20.8)$ & \\
\hline $\mathrm{T} 3 / \mathrm{T} 4$ & $30(4.2)$ & $12(9.2)$ & \\
\hline Lymph node status, n (\%) & & & $0.000^{\mathrm{a}}$ \\
\hline No & $553(76.8)$ & $80(6 \mid .5)$ & \\
\hline $\mathrm{NI} / 2$ & $167(23.2)$ & $50(38.5)$ & \\
\hline STAS, n (\%) & & & 0.156 \\
\hline Absent & $65 I(90.4)$ & $112(86.2)$ & \\
\hline Present & $69(9.6)$ & $18(13.8)$ & \\
\hline VPI, n (\%) & & & 0.525 \\
\hline Absent & $601(83.5)$ & $119(80.8)$ & \\
\hline Present & $105(80.8)$ & $25(19.2)$ & \\
\hline MVI, n (\%) & & & $0.001^{\mathrm{a}}$ \\
\hline Absent & $663(92.1)$ & $108(83.1)$ & \\
\hline Present & $57(7.9)$ & $22(16.9)$ & \\
\hline
\end{tabular}

Notes: Data are presented as $\mathrm{n}(\%)$. Group a: platelet $<300 \times 10^{\wedge} 9 / \mathrm{L}$, group b: platelet $\geq 300 \times 10^{\wedge} 9 / \mathrm{L}$ (two patients of which the platelet $<100 \times 10^{\wedge} 9 / \mathrm{L}$ were excluded). ${ }^{a}$ Statistically significant.

significant differences between group $\alpha$ and group $\beta$ in T stage $(\mathrm{P}=0.019)$, lymph node positivity $(\mathrm{P}<0.001)$, and the incidence of MVI $(P<0.001)$. In group $\alpha$, the rate of lymph node metastasis was $23.2 \%$, and in group $\beta$, it was $38.5 \%(\mathrm{P}<0.001)$. (Table 3$)$

Previous studies have only analyzed these indicators between patients in the platelet reference range and those with thrombocytosis; no previous study has examined clinicopathological factors in patients with platelet counts within the reference range. Therefore, to further investigate the relationship between platelet count and tumor behavior, we divided the patients into six groups based on the platelet count (group 1: platelet count $<150 \times 10^{9} / \mathrm{L}$, group 2 : platelet count $150-200 \times$ $10^{9} / \mathrm{L}$, group 3: platelet count $200-250 \times 10^{9} / \mathrm{L}$, group 4: platelet count $250-300 \times 10^{9} / \mathrm{L}$, group 5: platelet count $300-400 \times 10^{9} / \mathrm{L}$, and group 6: platelet count $\left.\geq 400 \times 10^{9} / \mathrm{L}\right)$. Our results showed that as the platelet count increased, the lymph node-positive rate $(\mathrm{P}<0.001)$ and the incidence of MVI $(\mathrm{P}<0.001)$ significantly increased. In addition, the rate of lymph node metastasis increased from group 1 to group 4 , despite these patients all having platelet counts within the reference range. In group 6, the rate of lymph node metastasis reached $53.3 \%$. However, no significant difference was observed between the platelet count groups in $\mathrm{T}$ stage $(\mathrm{P}=0.158)$ or the incidence of STAS $(\mathrm{P}=$ 0.184) (Table 4).

Table 4 The Influence of Different Platelet Counts on Microscopic Vessel Invasion (MVI) and Lymph Nodes Metastasis

\begin{tabular}{|c|c|c|c|c|c|c|c|}
\hline PLT Group & $I(n=33)$ & $2(n=170)$ & $3(n=3 \mid 3)$ & $4(n=206)$ & $5(n=|| 5)$ & $6(n=15)$ & $P$ value \\
\hline $\begin{array}{l}\text { T stage, } \mathrm{n}(\%) \\
\text { TI } \\
\text { T2 } \\
\text { T3/T4 }\end{array}$ & $\begin{array}{l}25(75.8) \\
7(2 I .2) \\
I(3.0)\end{array}$ & $\begin{array}{l}139(81.8) \\
26(15.3) \\
5(2.9)\end{array}$ & $\begin{array}{l}252(80.5) \\
50(16.0) \\
I I(3.5)\end{array}$ & $\begin{array}{l}156(75.7) \\
37(18.0) \\
13(6.3)\end{array}$ & $\begin{array}{l}83(72.2) \\
22(19.1) \\
10(8.7)\end{array}$ & $\begin{array}{l}8(53.3) \\
5(33.3) \\
2(13.3)\end{array}$ & 0.131 \\
\hline $\begin{array}{l}\text { Lymph node status, n (\%) } \\
\text { No } \\
\text { NI/2 }\end{array}$ & $\begin{array}{l}29(87.9) \\
4(12.1)\end{array}$ & $\begin{array}{l}140(82.4) \\
30(17.6)\end{array}$ & $\begin{array}{l}242(77.3) \\
71(32.7)\end{array}$ & $\begin{array}{l}144(69.9) \\
62(30.1)\end{array}$ & $\begin{array}{l}73(63.5) \\
42(36.5)\end{array}$ & $\begin{array}{l}7(46.7) \\
8(53.3)\end{array}$ & $0.000^{\mathrm{a}}$ \\
\hline $\begin{array}{l}\text { STAS, n (\%) } \\
\text { Absent } \\
\text { Present }\end{array}$ & $\begin{array}{l}33 \\
0\end{array}$ & $\begin{array}{l}154(90.6) \\
16(9.4)\end{array}$ & $\begin{array}{l}28 I(89.8) \\
32(10.2)\end{array}$ & $\begin{array}{l}185(89.8) \\
21(10.2)\end{array}$ & $\begin{array}{l}100(87) \\
15(13)\end{array}$ & $\begin{array}{l}12(80) \\
3(20)\end{array}$ & 0.265 \\
\hline $\begin{array}{c}\text { VPI, n (\%) } \\
\text { Absent } \\
\text { Present }\end{array}$ & $\begin{array}{l}30(90.9) \\
3(9.1)\end{array}$ & $\begin{array}{l}150(88.2) \\
20(11.8)\end{array}$ & $\begin{array}{l}267(85.3) \\
46(31.9)\end{array}$ & $\begin{array}{l}156(75.7) \\
50(24.3)\end{array}$ & $\begin{array}{l}92(80) \\
23(20)\end{array}$ & $\begin{array}{l}13(86.7) \\
2(13.3)\end{array}$ & $0.012^{\mathrm{a}}$ \\
\hline $\begin{array}{c}\text { MVI, n (\%) } \\
\text { Absent } \\
\text { Present }\end{array}$ & $\begin{array}{l}33 \\
0\end{array}$ & $\begin{array}{l}163(95.9) \\
7(4.1)\end{array}$ & $\begin{array}{l}291(93.0) \\
22(7.0)\end{array}$ & $\begin{array}{l}178(86.4) \\
28(13.6)\end{array}$ & $\begin{array}{l}95(82.6) \\
20(17.4)\end{array}$ & $\begin{array}{l}13(86.7) \\
2(13.3)\end{array}$ & $0.000^{\mathrm{a}}$ \\
\hline
\end{tabular}

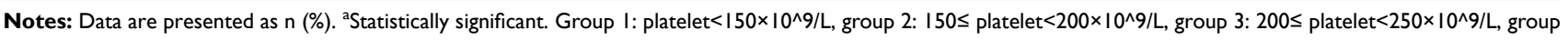

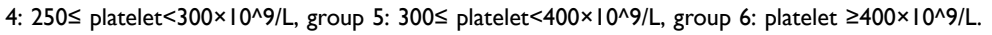




\section{The Influence of Different Subtypes on Platelet Count}

As the predominant subtype of ADC influences tumor behavior, such as lymph node metastasis, and poor prognosis, we investigated the relationships between subtype and platelet counts. We found that the platelet count in those with the solid-predominant subtype $\left(269.70 \pm 69.38 \times 10^{9} / \mathrm{L}\right)$ was significantly higher than those in patients with other subtypes (lepidic: $235.86 \pm 4.611 \times 10^{9} / \mathrm{L}$, acinar: $240.36 \pm 2.749 \times$ $10^{9} / \mathrm{L}$, papillary: $234.52 \pm 5.883 \times 10^{9} / \mathrm{L}$, micropapillary: $231.17 \pm 10.451 \times 10^{9} / \mathrm{L}$, and invasive mucinous adenocarcinoma: $\left.242.95 \pm 10.761 \times 10^{9} / \mathrm{L} ; \mathrm{P}<0.001\right)$. The solidpredominant subtype also had the second-highest lymph node-positive rate $(54.7 \% ; \mathrm{P}<0.001)$ and the highest incidence of MVI (23.3\%; P < 0.001) (Table 5). We further compared the solid-minor components group (patients with more than $5 \%$ solid subtype but with a predominant subtype other than solid; $\mathrm{n}=52$ ) and the solid-predominant group ( $\mathrm{n}$ =86) (Table 6). The platelet count in the solid-predominant group was significantly higher than that in the solid-minor components group $\left(269.70 \pm 69.378 \times 10^{9} / \mathrm{L}\right.$ vs $238.35 \pm$ $\left.58.473 \times 10^{9} / \mathrm{L} ; \mathrm{P}<0.001\right)$. We observed similar results in the lymph node-positive rate $(54.7 \%$ vs $22.2 \%$; $\mathrm{P}<0.001)$ and the incidence of MVI $(23.3 \%$ vs $7.7 \%$; $\mathrm{P}<0.001)$.

\section{Discussion}

Here, we investigated the possible correlation between platelet counts and lymph node metastasis in 852 lung invasive ADC cases. Thrombocytosis was significantly related to
Table 6 The Influence of Different Contents of Solid Subtypes on Platelet Counts

\begin{tabular}{|c|c|c|c|}
\hline Subtypes & $\begin{array}{l}\text { Solid-Minor } \\
\text { Components }(n=52)\end{array}$ & $\begin{array}{l}\text { Solid- } \\
\text { Predominant } \\
(n=86)\end{array}$ & $P$ value \\
\hline PLT $\left(\times 10^{\wedge} 9 / \mathrm{L}\right)$ & $238.35 \pm 58.473$ & $269.70 \pm 69.378$ & $0.000^{\mathrm{a}}$ \\
\hline $\begin{array}{l}\text { Lymph node } \\
\text { status, n (\%) } \\
\text { N0 } \\
\text { NI/2 }\end{array}$ & $\begin{array}{l}596(77.8) \\
170(22.2)\end{array}$ & $\begin{array}{l}39(45.3) \\
47(54.7)\end{array}$ & $0.000^{\mathrm{a}}$ \\
\hline $\begin{array}{l}\text { STAS, n (\%) } \\
\text { Absent } \\
\text { Present }\end{array}$ & $\begin{array}{l}690(90.1) \\
76(9.9)\end{array}$ & $\begin{array}{l}75(87.2) \\
\mathrm{II}(12.8)\end{array}$ & 0.405 \\
\hline $\begin{array}{c}\text { VPI, n (\%) } \\
\text { Absent } \\
\text { Present }\end{array}$ & $\begin{array}{l}642(83.8) \\
124(16.2)\end{array}$ & $\begin{array}{l}66(76.7) \\
20(23.3)\end{array}$ & $0.097^{\mathrm{a}}$ \\
\hline $\begin{array}{c}\text { MVI, n (\%) } \\
\text { Absent } \\
\text { Present }\end{array}$ & $\begin{array}{l}707(92.3) \\
59(7.7)\end{array}$ & $\begin{array}{l}66(76.7) \\
20(23.3)\end{array}$ & $0.000^{\mathrm{a}, \mathrm{b}}$ \\
\hline
\end{tabular}

Notes: Data are presented as $n(\%)$ or means \pm SD. ${ }^{a}$ Statistically significant. ${ }^{b}$ Fisher's exact test.

lymph node metastasis in lung ADC patients. In addition, higher platelet counts within the reference range were also associated with increased lymph node metastasis. Further, patients with the solid-predominant subtype had significantly higher platelet counts than patients with other pathological subtypes. We speculate that this may be one of the causes of the higher rate of lymph node metastasis in patients with the solid-predominant subtype.

Table 5 The Influence of Different Predominant Subtype on Platelet Count

\begin{tabular}{|c|c|c|c|c|c|c|c|}
\hline Subtype & $L(n=\mid 48)$ & $A(n=420)$ & $P(n=\mid 28)$ & $M P(n=23)$ & $S(n=84)$ & IMA (n=39) & $P$ value \\
\hline PLT (×10^9/L) & $235.86 \pm 4.6 \mathrm{II}$ & $240.36 \pm 2.749$ & $234.52 \pm 5.883$ & $231.17 \pm 10.45 \mid$ & $269.70 \pm 7.570$ & $242.95 \pm 10.761$ & 0.000 \\
\hline $\begin{array}{l}\text { Lymph node status } \\
\text { N0, n (\%) } \\
\text { NI/2, n (\%) }\end{array}$ & $\begin{array}{l}\mid 44(97.3) \\
4(2.7)\end{array}$ & $\begin{array}{l}317(74.4) \\
109(25.6)\end{array}$ & $\begin{array}{l}100(77.5) \\
29(22.5)\end{array}$ & $\begin{array}{l}6(26.1) \\
17(73.9)\end{array}$ & $\begin{array}{l}39(45.3) \\
47(54.7)\end{array}$ & $\begin{array}{l}29(72.5) \\
\text { II (27.5) }\end{array}$ & 0.000 \\
\hline $\begin{array}{l}\text { STAS } \\
\text { Absent } \\
\text { Present }\end{array}$ & $\begin{array}{l}139(93.9) \\
9(6.1)\end{array}$ & $\begin{array}{l}387(90.8) \\
39(9.2)\end{array}$ & $\begin{array}{l}109(84.5) \\
20(15.5)\end{array}$ & $\begin{array}{l}20(87) \\
3(\mid 3)\end{array}$ & $\begin{array}{l}75(87.2) \\
\mathrm{II}(12.8)\end{array}$ & $\begin{array}{l}35(87.5) \\
5(12.5)\end{array}$ & $0.114^{\mathrm{a}}$ \\
\hline $\begin{array}{l}\text { VPI } \\
\text { Absent } \\
\text { Present }\end{array}$ & $\begin{array}{l}133(89.9) \\
15(10.1)\end{array}$ & $\begin{array}{l}360(84.5) \\
66(15.5)\end{array}$ & $\begin{array}{l}99(76.7) \\
30(23.3)\end{array}$ & $\begin{array}{l}19(82.6) \\
4(17.4)\end{array}$ & $\begin{array}{l}66(76.7) \\
20(23.3)\end{array}$ & $\begin{array}{l}3 I(77.5) \\
9(22.5)\end{array}$ & $0.023^{\mathrm{a}}$ \\
\hline $\begin{array}{l}\text { MVI } \\
\text { Absent } \\
\text { Present }\end{array}$ & $\begin{array}{l}145(98) \\
3(2)\end{array}$ & $\begin{array}{l}397(93.2) \\
29(6.8)\end{array}$ & $\begin{array}{l}110(85.3) \\
19(14.7)\end{array}$ & $\begin{array}{l}18(78.3) \\
5(21.7)\end{array}$ & $\begin{array}{l}66(76.7) \\
20(23.3)\end{array}$ & $\begin{array}{l}37(92.5) \\
3(7.5)\end{array}$ & $0.000^{\mathrm{a}}$ \\
\hline
\end{tabular}

Notes: Data are presented as $\mathrm{n}(\%)$ or means \pm SEM. ${ }^{\text {aS }}$ tatistically significant. 
There are complex interactions between tumor cells and platelets. However, the specific mechanism of their interaction is still under study. Malignancies may promote the production of platelets in a variety of ways, ${ }^{31-33}$ and platelets can affect tumor behavior in return. Platelets can promote tumor cell migration and invasion into the surrounding microenvironment, as well as facilitating tumor arrest at the endothelium, extravasation, and seeding. ${ }^{34-36}$ They can also protect tumor cells from NK cell-mediated lysis. ${ }^{37,38}$ Several studies have reported that platelet depletion results in reduced average tumor weight in patients receiving chemotherapy. Conversely, platelet transfusion increases average aggregate tumor weight. ${ }^{39-41}$ Recent evidence suggest that increases in platelets and megakaryocytes can also inhibit metastasis in a site-specific manner. ${ }^{23}$ However, these findings were only in preclinical studies in breast cancer. Based on our results, we can conclude that an increase in the platelet count, even within the reference range, is associated with lymph node metastasis in lung ADC patients.

Higher platelet levels indicate lymph node metastasis in patients with invasive ADC. ${ }^{32,33}$ We examined platelet levels based on histological subtype and found that the platelet count and the incidence of MVI in patients with solid-predominant tumors were significantly higher than in patients with other histologies. Further, the rate of lymph node metastasis increased with increased platelet count, even if the platelet count was within the reference range. In group $\alpha$ (platelet count: $100-300 \times 10^{9} / \mathrm{L}$ ), the rate of lymph node metastasis was $23.2 \%$, whereas it was $38.5 \%$ in group $\beta$ (platelet count: $>300 \times 10^{9} / \mathrm{L} ; \mathrm{P}<0.001$ ). In patients with a platelet count $>400 \times 10^{9} / \mathrm{L}$, the rate of lymph node metastasis was $53.3 \%$. The rates of lymph node metastasis in the four groups with platelet counts above $200 \times 10^{9} / \mathrm{L}$ were almost twice as high as the rates in the two groups with platelet counts below $200 \times$ $10^{9} / \mathrm{L}$. Groups $\alpha$ and $\beta$ also differed significantly in terms of $\mathrm{T}$ stage (Table 3 ). However, there was no significant difference in $\mathrm{T}$ stage among groups 1,2,3, and 4 (all within the reference range; Table 4). However, there was a significant difference in $\mathrm{N}$ stage. As the two main parameters for tumor growth, $\mathrm{T}$ stage and $\mathrm{N}$ stage are the most likely to be directly related to elevated platelets counts. Therefore, we can conclude that platelet count may have a direct relationship with lymph node metastasis, at least in patients with platelet counts in the reference range.

The relationship between elevated platelet counts and lymph node metastasis is not fully understood. Platelets are known to contain many angiogenic factors, such as vascular endothelial growth factor (VEGF) and platelet-derived growth factor (PDGF). VEGFs are present in resting platelets, which are then released on activation, ${ }^{42,43}$ and cancer cells can stimulate the secretion of VEGF-A and other proangiogenic factors from platelets. ${ }^{44}$ VEGF-C and VEGF-D facilitate tumor metastasis via modulating the lymphatic vessels, ${ }^{45-47}$ and VEGF-C can induce sentinel node lymphatic angiogenesis and promote lymphatic metastasis even before the tumor has metastasized. ${ }^{47,48}$ In addition, PDGF can stimulate the production of VEGFs by fibroblasts, which can expand the lymphatic vasculature. ${ }^{47,49,50} \mathrm{We}$ hypothesize that platelets may promote lymphatic metastasis of lung ADC tumor cells in a similar fashion. Some preclinical studies have shown that the exit routes provide by lymph node blood vessels may play role in metastatic tumor cell dissemination. ${ }^{51}$ Further, VEGF-C and VEGFR3 are related to lymphangiogenesis and the development of non-small cell lung cancer. ${ }^{52}$ However, as VEGF-C can influence megakaryopoiesis, the correlation of platelet counts and lymph node metastasis may be indirect. ${ }^{53}$

We found that patients with the solid-predominant subtype had the highest platelet counts. Therefore, we speculate that the increased platelets in the solid-predominant subtype may facilitate the increased lymphatic metastasis we observed. Tumor-induced lymphangiogenesis via VEGF-C/VEGFR-3 is the major mechanism through which metastasis to the lymph nodes is promoted. ${ }^{54}$ It is therefore very likely that the VEGF-C level is increased locally or systemically in patients with solid-predominant subtypes. However, this has not been assessed. Those with the solid-predominant subtype have higher levels of laminin-5, ezrin, VEGF, and CD204-TAMs than other subtypes. ${ }^{55}$ Studies have proven that increases in these factors are significantly related to lymphatic metastasis and poor prognosis in those with resected non-small cell lung cancer. ${ }^{55,56}$ However, the effects of platelets in different lung ADC subtypes have not been studied. This is the first study to examine the relationship between platelets and subtypes in lung ADC.

There is some evidence to suggest that aspirin can decrease levels of PDGF and VEGF in the platelet releasate. ${ }^{57-60}$ However, previous studies focused on how antiplatelet therapy inhibits platelet-mediated angiogenesis rather than platelet-mediated lymphatic spread. Based on our results, we hypothesize that antiplatelet therapy may reduce lymph node metastasis in all lung ADC patients. Because lymphatic system metastasis is a common cause 
of treatment failure and death in postoperative patients with non-small cell lung cancer, ${ }^{61}$ targeted therapy against lymph node metastasis can play an important postoperative role. Therefore, antiplatelet therapy such as low-dose aspirin may be a potent direction for lung ADC treatment. Long-term follow-up studies have shown that regular use of aspirin reduces the long-term risk of several cancers, as well as the risk of distant metastasis. ${ }^{62-64}$ For lung cancer, the benefit of aspirin was only confirmed in ADC. ${ }^{25}$ Anticoagulants are effective for patients with venous thromboembolism. However, antiplatelet therapy may be effective in the lymphatic system because of its effect on the platelet releasate. ${ }^{44}$ In a large clinical trial, small doses of aspirin reduced the risk of distant metastases in many ADCs and the overall risk of fatal ADC in the trial population. ${ }^{62}$ Another study showed that aspirin can reduce mortality in older patients with several types of ADC. ${ }^{63}$ In 2016, the US Preventive Services Task Force recommended regular use of low-dose aspirin in some people with risk factors for colorectal cancer. ${ }^{65}$ A large retrospective study including more than 10 million people in South Korea confirmed that long-term use of low-dose aspirin was associated with a decreased risk of lung cancer. ${ }^{66}$ Recognition of the tumorpromoting effects of platelets emphasizes the role of antiplatelet therapy in the prevention and treatment of cancer. Nevertheless, identifying cancer patients who will benefit from antiplatelet therapy is still an important issue. The potential risks of antiplatelet therapy should be fully evaluated and investigated.

There are some limitations to this study. First, all patients had invasive lung ADC and had undergone resection. Therefore, patients who were unable to tolerate surgery were excluded. Second, the classification of lung ADC subtypes is still being studied, and non-dominant subtypes might still play a role in tumor biology. Third, pathological inspection of biopsy specimens cannot fully represent the true content of pathological subtypes. ${ }^{67}$ Fourth, as a retrospective study, selection bias and performance bias are inevitable. Future multicenter clinical studies with larger samples may validate our results.

\section{Conclusions}

Elevated platelet counts are significantly associated with a higher rate of lymph node metastasis, even if the platelet counts are within the reference range. Platelet counts were significantly higher in patients with solid-predominant histology than in patients with other histologies. In addition, VEGF-C may play an important role in lymphatic metastasis in patients with lung ADC. We hypothesize that antiplatelet therapy may reduce lymph node metastasis in lung ADC patients.

\section{Acknowledgments}

We are very grateful to our patients and all participants in the data collection. We would like to thank Editage (www. editage.cn) and Dr. Sun Zhenguo for English language editing.

\section{Funding}

This study was funded by National Natural Science Foundation of China (NO. 81672292, 81802397), Project funded by Jinan Science and Technology Bureau (NO. 2019GXRC051), Natural Science Foundation of Shandong Province (NO. ZR2017BH035), Taishan Scholar Program of Shandong Province (NO. ts201712087).

\section{Disclosure}

The authors report no conflicts of interest in this work.

\section{References}

1. Bray F, Ferlay J, Soerjomataram I, Siegel RL, Torre LA, Jemal A. Global cancer statistics 2018: GLOBOCAN estimates of incidence and mortality worldwide for 36 cancers in 185 countries. CA Cancer J Clin. 2018;68(6):394-424. doi:10.3322/caac.21492

2. Travis WD, Brambilla E, Noguchi M, et al. International Association for the Study of Lung Cancer/American Thoracic Society/European Respiratory Society International Multidisciplinary Classification of lung adenocarcinoma. $J$ Thorac Oncol. 2011;6(2):244-285. doi:10.1097/JTO.0b013e318206a221

3. Ujiie H, Kadota K, Chaft JE, et al. Solid predominant histologic subtype in resected stage $\mathrm{i}$ lung adenocarcinoma is an independent predictor of early, extrathoracic, multisite recurrence and of poor postrecurrence survival. J Clin Oncol. 2015;33(26):2877-2884. doi:10.1200/JCO.2015.60.9818

4. Zhang Y, Wang R, Cai D, et al. A comprehensive investigation of molecular features and prognosis of lung adenocarcinoma with micropapillary component. J Thorac Oncol. 2014;9(12):1772-1778.

5. Luo J, Wang R, Han B, et al. Solid predominant histologic subtype and early recurrence predict poor postrecurrence survival in patients with stage I lung adenocarcinoma. Oncotarget. 2017;8(4):7050-7058.

6. Hung -J-J, Yeh Y-C, Wu Y-C, Chou T-Y, Hsu W-H. Prognostic factors in completely resected node-negative lung adenocarcinoma of $3 \mathrm{~cm}$ or smaller. $J$ Thorac Oncol. 2017;12(12):1824-1833.

7. Xu S, Xi J, Jiang W, Lu S, Wang Q. Solid component and tumor size correlate with prognosis of stage IB lung adenocarcinoma. Ann Thorac Surg. 2015;99(3):961-967.

8. Tesfamariam B. Involvement of platelets in tumor cell metastasis. Pharmacol Ther. 2016;157:112-119.

9. Contursi A, Sacco A, Grande R, Dovizio M, Patrignani P. Platelets as crucial partners for tumor metastasis: from mechanistic aspects to pharmacological targeting. Cell Mol Life Sci. 2017;74(19):3491-3507.

10. Haemmerle M, Stone RL, Menter DG, Afshar-Kharghan V, Sood AK. The platelet lifeline to cancer: challenges and opportunities. Cancer Cell. 2018;33(6):965-983. doi:10.1016/j. ccell.2018.03.002 
11. Meikle CKS, Kelly CA, Garg P, Wuescher LM, Ali RA, Worth RG. Cancer and thrombosis: the platelet perspective. Front Cell Dev Biol. 2016;4:147.

12. Labelle M, Hynes RO. The initial hours of metastasis: the importance of cooperative host-tumor cell interactions during hematogenous dissemination. Cancer Discov. 2012;2(12):1091-1099. doi:10.1158/ 2159-8290.CD-12-0329

13. Franco AT, Corken A, Ware J. Platelets at the interface of thrombosis, inflammation, and cancer. Blood. 2015;126(5):582-588. doi:10.1182/ blood-2014-08-531582

14. Plantureux L, Mège D, Crescence L, Dignat-George F, Dubois C, Panicot-Dubois L. Impacts of cancer on platelet production, activation and education and mechanisms of cancer-associated thrombosis. Cancers. 2018;10(11):11. doi:10.3390/cancers 10110441

15. Xu XR, Zhang D, Oswald BE, et al. Platelets are versatile cells: new discoveries in hemostasis, thrombosis, immune responses, tumor metastasis and beyond. Crit Rev Clin Lab Sci. 2016;53(6):409-430.

16. Agoston AT, Srivastava A, Zheng Y, Bueno R, Odze RD, Szallasi Z. Paraneoplastic thrombocytosis is associated with increased mortality and increased rate of lymph node metastasis in oesophageal adenocarcinoma. Pathology. 2017;49(5):471-475. doi:10.1016/j.pathol.2017.04.001

17. Słabuszewska-Jóźwiak A, Dmoch-Gajzlerska E, Kozakiewicz B, Jakiel G. The prognostic significance of thrombocytosis in ovarian cancer. Ann Agric Environ Med. 2015;22(4):731-735. doi:10.5604/ 12321966.1185785

18. Harano K, Kogawa T, Wu J, et al. Thrombocytosis as a prognostic factor in inflammatory breast cancer. Breast Cancer Res Treat. 2017;166(3):819-832. doi:10.1007/s10549-017-4463-6

19. Shoda K, Komatsu S, Ichikawa D, et al. [Thrombocytosis associated with poor prognosis in patients with gastric cancer]. Gan to Kagaku Ryoho. 2015;42(12):1980-1982. Japanese.

20. Rao X-D, Zhang H, Xu Z-S, Cheng H, Shen W, Wang X-P. Poor prognostic role of the pretreatment platelet counts in colorectal cancer: A meta-analysis. Medicine. 2018;97(23):e10831. doi:10.1097/ MD.0000000000010831

21. Baranyai Z, Jósa V, Tóth A, et al. Paraneoplastic thrombocytosis in gastrointestinal cancer. Platelets. 2016;27(4):269-275. doi:10.3109/ 09537104.2016.1170112

22. Wang Y-H, Kang J-K, Zhi Y-F, et al. The pretreatment thrombocytosis as one of prognostic factors for gastric cancer: A systematic review and meta-analysis. Int $J$ Surg. 2018;53:304-311. doi:10.1016/ j.ijsu.2018.03.084

23. Foss A, Munoz-Sagredo L, Sleeman J, Thiele W. The contribution of platelets to intravascular arrest, extravasation, and outgrowth of disseminated tumor cells. Clin Exp Metastasis. 2020;37(1):47-67. doi:10.1007/s10585-019-10009-y

24. Wang -J-J, Wang Y-L, Ge -X-X, et al. Prognostic values of platelet-associated indicators in resectable lung cancers. Technol Cancer Res Treat. 2019;18:1533033819837261. doi:10.1177/1533033819837261

25. Li Y, Miao L-Y, Xiao Y-L, Cai H-R, Zhang D-P. Elevated platelets enhance cancer cell migration, promote hematogenous metastasis and associate with a poor prognosis in advanced non-small cell lung cancer cases. Asian Pac J Cancer Prev. 2014;15(1):139-143. doi:10.7314/APJCP.2014.15.1.139

26. Huang H, Wang T, Hu B, Pan C. Visceral pleural invasion remains a size-independent prognostic factor in stage I non-small cell lung cancer. Ann Thorac Surg. 2015;99(4):1130-1139. doi:10.1016/j. athoracsur.2014.11.052

27. Neri S, Yoshida J, Ishii G, et al. Prognostic impact of microscopic vessel invasion and visceral pleural invasion in non-small cell lung cancer: a retrospective analysis of 2657 patients. Ann Surg. 2014;260 (2):383-388. doi:10.1097/SLA.0000000000000617

28. Shimada Y, Saji H, Kato Y, et al. The frequency and prognostic impact of pathological microscopic vascular invasion according to tumor size in non-small cell lung cancer. Chest. 2016;149 (3):775-785.
29. Vaghjiani RG, Takahashi Y, Eguchi T, et al. Tumor spread through air spaces is a predictor of occult lymph node metastasis in clinical stage ia lung adenocarcinoma. $J$ Thorac Oncol. 2020;15(5):792-802. doi:10.1016/j.jtho.2020.01.008

30. Song T, Jiang L, Zhuo Z, et al. Impacts of thoracoscopic surgery and high grade histologic subtypes on spread through air spaces in small stage I lung adenocarcinomas. J Cancer Res Clin Oncol. 2019;145 (9):2375-2382. doi:10.1007/s00432-019-02972-6

31. Long Y, Wang T, Gao Q, Zhou C. Prognostic significance of pretreatment elevated platelet count in patients with colorectal cancer: a meta-analysis. Oncotarget. 2016;7(49):81849-81861. doi:10.18632/ oncotarget. 13248

32. Gao L, Zhang H, Zhang B, Zhang L, Wang C. Prognostic value of combination of preoperative platelet count and mean platelet volume in patients with resectable non-small cell lung cancer. Oncotarget. 2017;8(9):15632-15641. doi:10.18632/oncotarget.14921

33. Liu HB, Gu XL, Ma XQ, et al. Preoperative platelet count in predicting lymph node metastasis and prognosis in patients with non-small cell lung cancer. Neoplasma. 2013;60(2):203-208.

34. Xu XR, Yousef GM, Ni H. Cancer and platelet crosstalk: opportunities and challenges for aspirin and other antiplatelet agents. Blood. 2018;131(16):1777-1789.

35. Lin RJ, Afshar-Kharghan V, Schafer AI. Paraneoplastic thrombocytosis: the secrets of tumor self-promotion. Blood. 2014;124 (2):184-187. doi:10.1182/blood-2014-03-562538

36. Pucci F, Rickelt S, Newton AP, et al. PF4 Promotes platelet production and lung cancer growth. Cell Rep. 2016;17(7):1764-1772.

37. Placke T, Örgel M, Schaller M, et al. Platelet-derived MHC class I confers a pseudonormal phenotype to cancer cells that subverts the antitumor reactivity of natural killer immune cells. Cancer Res. 2012;72(2):440-448. doi:10.1158/0008-5472.CAN-11-1872

38. Palacios-Acedo AL, Mège $\mathrm{D}$, Crescence L, Dignat-George F, Dubois C, Panicot-Dubois L. Platelets, thrombo-inflammation, and cancer: collaborating with the enemy. Front Immunol. 2019;10:1805. doi:10.3389/fimmu.2019.01805

39. Demers M, Ho-Tin-Noé B, Schatzberg D, Yang JJ, Wagner DD. Increased efficacy of breast cancer chemotherapy in thrombocytopenic mice. Cancer Res. 2011;71(5):1540-1549. doi:10.1158/00085472.CAN-10-2038

40. Bottsford-Miller J, Choi H-J, Dalton HJ, et al. Differential platelet levels affect response to taxane-based therapy in ovarian cancer. Clin Cancer Res. 2015;21(3):602-610. doi:10.1158/1078-0432.CCR-140870

41. Huong PT, Nguyen LT, Nguyen X-B, Lee SK, Bach D-H. The role of platelets in the tumor-microenvironment and the drug resistance of cancer cells. Cancers. 2019;11:2.

42. Lim L, Bui H, Farrelly O, et al. Hemostasis stimulates lymphangiogenesis through release and activation of VEGFC. Blood. 2019;134 (20):1764-1775.

43. Chatterjee M, Huang Z, Zhang W, et al. Distinct platelet packaging, release, and surface expression of proangiogenic and antiangiogenic factors on different platelet stimuli. Blood. 2011;117 (14):3907-3911.

44. Battinelli EM, Markens BA, Italiano JE. Release of angiogenesis regulatory proteins from platelet alpha granules: modulation of physiologic and pathologic angiogenesis. Blood. 2011;118 (5):1359-1369.

45. Karpanen T, Egeblad M, Karkkainen MJ, et al. Vascular endothelial growth factor $\mathrm{C}$ promotes tumor lymphangiogenesis and intralymphatic tumor growth. Cancer Res. 2001;61(5):1786-1790.

46. Tammela T, Alitalo K. Lymphangiogenesis: molecular mechanisms and future promise. Cell. 2010;140(4):460-476.

47. Hirakawa S, Brown LF, Kodama S, Paavonen K, Alitalo K, Detmar M. VEGF-C-induced lymphangiogenesis in sentinel lymph nodes promotes tumor metastasis to distant sites. Blood. 2007;109 (3):1010-1017. 
48. Harrell MI, Iritani BM, Ruddell A. Tumor-induced sentinel lymph node lymphangiogenesis and increased lymph flow precede melanoma metastasis. Am J Pathol. 2007;170(2):774-786.

49. Hirakawa S, Kodama S, Kunstfeld R, Kajiya K, Brown LF, Detmar M. VEGF-A induces tumor and sentinel lymph node lymphangiogenesis and promotes lymphatic metastasis. J Exp Med. 2005;201(7):1089-1099.

50. Shikada Y, Yonemitsu Y, Koga T, et al. Platelet-derived growth factor-AA Is an essential and autocrine regulator of vascular endothelial growth factor expression in non-small cell lung carcinomas. Cancer Res. 2005;65(16):7241.

51. Brown M, Assen FP, Leithner A, et al. Lymph node blood vessels provide exit routes for metastatic tumor cell dissemination in mice. Science. 2018;359(6382):1408-1411.

52. Li Q, Dong X, Gu W, Qiu X, Wang E. Clinical significance of co-expression of VEGF-C and VEGFR-3 in non-small cell lung cancer. Chin Med J. 2003;116(5):727-730.

53. Thiele W, Krishnan J, Rothley M, et al. VEGFR-3 is expressed on megakaryocyte precursors in the murine bone marrow and plays a regulatory role in megakaryopoiesis. Blood. 2012;120 (9):1899-1907.

54. Su JL, Yen CJ, Chen PS, et al. The role of the VEGF-C/VEGFR-3 axis in cancer progression. Br J Cancer. 2007;96(4):541-545.

55. Saruwatari K, Ikemura S, Sekihara K, et al. Aggressive tumor microenvironment of solid predominant lung adenocarcinoma subtype harboring with epidermal growth factor receptor mutations. Lung Cancer. 2016;91.

56. Naito M, Aokage K, Saruwatari K, et al. Microenvironmental changes in the progression from adenocarcinoma in situ to minimally invasive adenocarcinoma and invasive lepidic predominant adenocarcinoma of the lung. Lung Cancer. 2016;100:53-62.

57. Nadar S, Blann AD, Lip GYH. Effects of aspirin on intra-platelet vascular endothelial growth factor, angiopoietin-1, and p-selectin levels in hypertensive patients. Am J Hypertens. 2006;19:9.
58. Holmes CE, Jasielec J, Levis JE, Skelly J, Muss HB. Initiation of aspirin therapy modulates angiogenic protein levels in women with breast cancer receiving tamoxifen therapy. Clin Transl Sci. 2013;6 (5):386-390.

59. Sandset PM. CXCL4-platelet factor 4, heparin-induced thrombocytopenia and cancer. Thromb Res. 2012;129(Suppl 1):S97-S100.

60. de Winter P, Leoni P, Abraham D. Connective tissue growth factor: structure-function relationships of a mosaic, multifunctional protein. Growth Factors. 2008;26(2):80-91.

61. Hung -J-J, Yeh Y-C, Jeng W-J, et al. Prognostic factors of survival after recurrence in patients with resected lung adenocarcinoma. J Thorac Oncol. 2015;10(9):1328-1336.

62. Algra AM, Rothwell PM. Effects of regular aspirin on long-term cancer incidence and metastasis: a systematic comparison of evidence from observational studies versus randomised trials. Lancet Oncol. 2012;13(5):518-527.

63. Loomans-Kropp HA, Pinsky P, Cao Y, Chan AT, Umar A. Association of Aspirin use with mortality risk among older adult participants in the prostate, lung, colorectal, and ovarian cancer screening trial. JAMA Netw Open. 2019;2(12):e1916729.

64. Lichtenberger LM, Vijayan KV. Are Platelets the primary target of aspirin's remarkable anticancer activity? Cancer Res. 2019;79 (15):3820-3823.

65. Bibbins-Domingo K, Grossman DC, Curry SJ, et al. Screening for colorectal cancer: US Preventive Services Task Force Recommendation Statement. JAMA. 2016;315(23):2564-2575.

66. Ye S, Lee M, Lee D, Ha E-H, Chun EM. Association of long-term use of low-dose aspirin as chemoprevention with risk of lung cancer. JAMA Netw Open. 2019;2(3):e190185.

67. Wright J, Churg A, Kitaichi M, Yang H-M, Hyde D, Yi E. Reproducibility of visual estimation of lung adenocarcinoma subtype proportions. Mod Pathol. 2019;32(11):1587-1592.

\section{Publish your work in this journal}

Cancer Management and Research is an international, peer-reviewed open access journal focusing on cancer research and the optimal use of preventative and integrated treatment interventions to achieve improved outcomes, enhanced survival and quality of life for the cancer patient.
The manuscript management system is completely online and includes a very quick and fair peer-review system, which is all easy to use. Visit http://www.dovepress.com/testimonials.php to read real quotes from published authors. 